http://jmscr.igmpublication.org/home/ ISSN (e)-2347-176x ISSN (p) 2455-0450

crossref DOI: https://dx.doi.org/10.18535/jmscr/v7i10.36

\author{
Journal Of Medical Science And Clinical Research \\ IGM Publication \\ An Official Publication of IGM Publication
}

\title{
Application of damage control orthopaedics in the management of type III open tibia fractures in a hilly region of North India
}

\author{
Authors \\ Tanveer Ahmed Bhat ${ }^{1}$, Imtiyaz Ahmad Beigh ${ }^{2 *}$, Sayar Ahmad Mantoo ${ }^{3}$, \\ Malik Naseer Ahmad ${ }^{4}$, Zubair Ahmad Lone ${ }^{5}$, Tanveer Ali ${ }^{6}$, Karanbir Singh ${ }^{7}$ \\ ${ }^{1,2,3}$ Senior Resident, SGRRIM \& HS, Dehradun \\ ${ }^{4}$ Medical Officer, Deptt. of Health Medical Education, J\&K \\ ${ }^{5,6,7}$ PG III Yr, Department of Orthopaedics, Govt Medical College, Jammu \\ *Corresponding Author \\ Dr Imtiyaz Ahmad Beigh \\ Senior Resident, SGRRIM \& HS, Dehradun, India
}

\begin{abstract}
Introduction: This study was conducted to analyse the outcomes of type III open tibia fractures arising out of high energy motor vehicle accidents in the hilly areas of Jammu province managed by applying the principles of damage control orthopaedics.

Methods: A retrospective study was carried out in seventeen patients with type III open tibia fractures. These patients were selected among the 243 cases that were injured in five major MVAs in the hilly areas of Jатти province between 2016 and 2019. External fixators were used for stabilisation of fractures in all patients on the first day of admission in Govt. Medical College and Hospital, Jammu according to principles of damage control orthopaedics.

Results: Bone union was achieved in fourteen patients. One patient needed amputation on the fifth postop day. The mean time for bone union was 2.9 months (2 to 3.5 months) in case of conversion to internal fixation, and 6.5 months (2 to 15 months) when external fixation was extended and used as definitive method of fixation. No infectious complications were observed in the cases which underwent early conversion definitive fixation. After a mean follow-up of 24 months (7 to 30 months) fourteen patients recovered functionally and thirteen could return to duty with one patient still recovering from sciatic nerve injury.

Conclusion: This study highlights the utility of temporary external fixation as a damage control treatment modality in both preventing a second- hit phenomenon and enhancing bone healing and functional recovery in type III open tibia fractures.

Keywords: Damage control orthopaedics; External fixator; Tibia fracture; Motor Vehicle Accident; Open fracture.
\end{abstract}

\section{Introduction}

According to AAOS: Damage Control Orthopaedics (DCO) refers to limited early surgical intervention for stabilisation of musculoskeletal injuries in the unstable polytrauma patient. By decreasing operative times and tissue handling the lethal triad of coagulopathy, acidosis and hypothermia is prevented. It avoids the second hit of an extensive definitive procedure and minimises initial 
morbidity ${ }^{1}$. Motor vehicle accidents (MVAs) cause around 1.2 million deaths per year globally ${ }^{2}$. In the hilly region of Jammu, poorly developed national highways along with inefficient enforcement of road traffic laws lead to increased incidence of MVAs. Most of the times buses carrying hundreds of passengers fall into deep gorges which lead to casualities on a large scale necessitating medical evacuation by aircrafts.

\section{Patients and Methods}

A retrospective study was conducted in Government Medical College and Hospital, Jammu (India) in which seventeen patients of mean age 28 years (range: 20 to 51 years), having open type III tibia fractures resulting from bus crashes from hilly roads, injured between 2016 and 2019 were included. There were 8 type IIIa fractures, 6 type IIIb fractures and 3 type IIIc fractures. All patients required a DCO procedure with external fixation on admission. Ten patients

Table 1: Patient Details had associated injuries, five among those had polytrauma with Injury Severity Score of > 16. External fixation was extended to an average period of 10.4 weeks (range: 8 to 13 weeks) in five cases before converting into ORIF either because of fracture complexity or soft tissue damage and infection. Reamed intramedullary interlocking nailing was done in 8 cases after removing external fixators at an average period of 5.2 days (range: 3 to 7 days). Two patients had to be converted from mono-planar external fixation to ring fixators. One patient had mono-planar external fixator removed at 6 weeks and Taylor Spatial Frame was applied and continued for 24 weeks. Bone morphogenetic proteins (BMPs) were not used in any case. There were 8 type IIIa fractures, 6 type IIIb fractures and 3 type IIIc fractures. Twelve patients were males and five were females, with a mean age of 28 years $(20$ 51 years).

\begin{tabular}{|c|c|c|c|}
\hline CASE & AGE & BONE INJURIES & ASSOCIATED INJURIES \\
\hline 1 & 31 & $\begin{array}{l}\text { Type IIIa open tibia fracture }(\mathrm{R}) \text {; closed frac. Shaft } \\
\text { humerus }(\mathrm{R})\end{array}$ & ----- \\
\hline 2 & 53 & Type IIIb open tibia fracture(L) & Partial section of deep peroneal nerve \\
\hline 3 & 37 & Type IIIb open tibia fracture $(\mathrm{R})$ & Polytrauma \\
\hline 4 & 39 & Type IIIa open tibia fracture(L) & Sciatic nerve injury \\
\hline 5 & 20 & $\begin{array}{l}\text { Type IIIc open tibia fracture(R); Open fract. distal } 1 / 3 \\
\text { femur }\end{array}$ & $\begin{array}{l}\text { Posterior tibial artery injury; Heelpad } \\
\text { avulsion }\end{array}$ \\
\hline 6 & 23 & $\begin{array}{l}\text { Closed frac, middle } 1 / 3 \text {; Femur( R); Type IIIc tibia } \\
\text { fracture(R) }\end{array}$ & Sup. Femoral artery injury \\
\hline 7 & 23 & Type IIIc open tibia fracture(L) & Section of the posterior tibial artery \\
\hline 8 & 30 & $\begin{array}{l}\text { Open fract. proximal } 1 / 3 \text { of the radius }(\mathrm{R}) \text {; Type IIIb } \\
\text { open tibia fracture }(\mathrm{R})\end{array}$ & Polytrauma; Section of radial nerve \\
\hline 9 & 36 & Type IIIc open tibia fracture(L); Hip dislocation (R) & Polytrauma \\
\hline 10 & 24 & Type IIIa open tibia fracture(L) & ----- \\
\hline 11 & 32 & Type IIIa open tibia fracture $(\mathrm{R})$ & ----- \\
\hline 12 & 31 & $\begin{array}{l}\text { Open femoral fracture }(\mathrm{R}) ; \text { Type IIIb open tibia } \\
\text { fracture }(\mathrm{R})\end{array}$ & Common peroneal nerve injury \\
\hline 13 & 27 & $\begin{array}{l}\text { Closed fract. middle } 1 / 3 \text { of the Femur }(\mathrm{R}) ; \text { Type IIIa } \\
\text { open tibia fracture }(\mathrm{R})\end{array}$ & Polytrauma \\
\hline 14 & 23 & Type IIIa open tibia fracture(L) & - \\
\hline 15 & 29 & Type IIIa open tibia fracture(R) & - \\
\hline 16 & 27 & Type IIIa open tibia fracture(L) & ---- \\
\hline 17 & 55 & Type IIIb open tibia fracture (R) & Polytrauma \\
\hline
\end{tabular}

\section{Resuscitation and Polytrauma Management}

Hemodynamic stability of all patients was achieved in the emergency room by administration of intravenous crystalloid fluids and whole blood. When replacement of fluid and blood did not stabilise the patient's vital signs, additional steps were taken. Among the five polytrauma patients two had head injuries, one of 
which required hematoma evacuation. Two polytrauma patients with massive hemothorax required chest tube drainage. One patient had hemoperitoneum with grade 5 splenic injury and had splenectomy done.

\section{Damage Control}

All the fractures were treated by early and thorough wound debridement and the application of an external fixator in emergency operating room. Thefixator construct used was selected depending on the type of fracture and the extent and location of the soft-tissue injury. Prophylactic cefuroxime 1.5 grams B.D. and amikacin $500 \mathrm{mg}$ B.D. was used in all cases. Plastic surgeon consultation was sought on the same day and they performed all flap coverage procedures. Vascular surgeons were consulted in all type IIIc fractures and reverse saphenous grafts were used to repair arterial injuries. The duration of external fixation was based on the state of the soft tissues and the extent of bone union.

\section{Type IIIa Fractures}

There were 8 type 1Ila fractures. Temporary external fixation was used in all cases. Conversion to internal fixation was performed within a mean period of 8 days after trauma. Mono-planar external fixators were used in 5 cases and deltatype tibio-calcaneal external fixators were used in 3 cases. Of these, three cases required bone grafting and one required flap cover of the skin defect prior to definitive fixation. Case no. 1 with closed fracture of the humeral shaft was plated on day five after removal of external fixation. Case 13 with associated closed fracture of middle onethird of femur was converted into locked intramedullary nail on the seventh day. The mean hospitalisation time of this group was 4 weeks and one multiply injured patient (case 13) was hospitalised for 9 weeks. Return to work was at an average of 32.5 weeks after the fracture.

\section{Type IIIb Fractures}

There were 5 type IIIb open tibia fractures. Temporary external fixation was used in all cases. Three patients had polytrauma (cases 3, 8, 17). Two cases with polytrauma developed fat embolism syndrome within a 5-day period and required ICU care. Case no.8 required rotational medial gastrocnemius flap cover whereas case 17 required lateral gastrocnemius rotational flap and skin-grafting to cover exposed tibia. Case 2, 3 and 12 required SSG. In five cases external fixator was maintained for a mean duration of 10.4 weeks before final conversion to open reduction and internal fixation. All cases required corticocancellous bone grafting. Three patients showed signs of pin-site discharge or local erythema and needed multiple debridements. The mean hospitalisation time was 80 days. Return to work was after an average of 63.7 weeks.

\section{Type IIIc Fractures}

There were four type IIIc fractures. Case no. 5 was initially fixed with delta -type tibio-calcaneal external fixator and posterior tibial artery injury was repaired on day-one by reverse saphenous venous graft. Later this patient developed gangrenous changes in leg and foot and on day-5 this patient required amputation. Case no. 6 had injury of superficial femoral artery along with associated fracture shaft of femur. Arterial repair was done by vascular surgeon using reverse saphenous venous graft and then a knee- spanning external fixator was used for temporary fixation of femoral and tibia fractures. Patient had exposed tibia and required a lateral gastrocnemius flap. At 6 weeks this was converted to a Taylor Spatial frame which was continued for 22 weeks. Case no. 7 had posterior tibial artery injury just above ankle for which vascular surgeon consultation was sought who decided not to intervene as dorsalis pedis artery was intact. A delta fixator was initially applied which was converted to anIlizarov ring fixator at 5 weeks and removed at 24 weeks. Patient also required split- thickness 


\section{JMSCR Vol||07||Issue||10||Page 199-205||October}

skin grafting. Case no. 9 had associated posterior hip dislocation and injury of superficial femoral artery. Emergent reduction of hip dislocation was done in the emergency room under sedation using East Baltimore lift maneuver. Vascular repair was done using reverse saphenous graft. Kneespanning external fixator was used to stabilize fracture as also to prevent tensioning of vascular anastomosis. It was converted into ring fixator at 5 weeks but had no evidence of healing at 6 months. Cancellous bone grafting was used twice in this case but still failed to unite at last follow-up at 20 months.

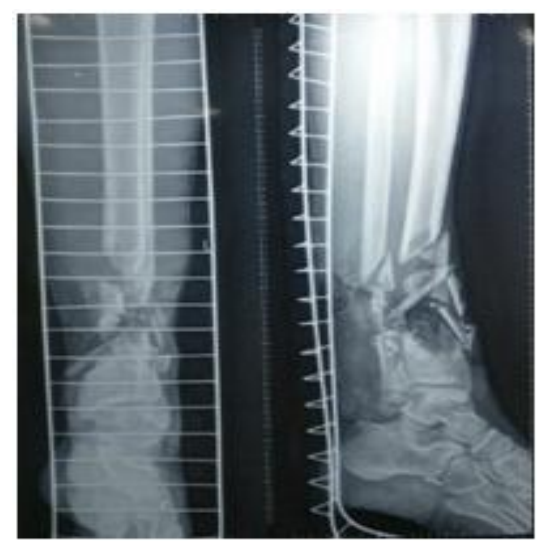

A

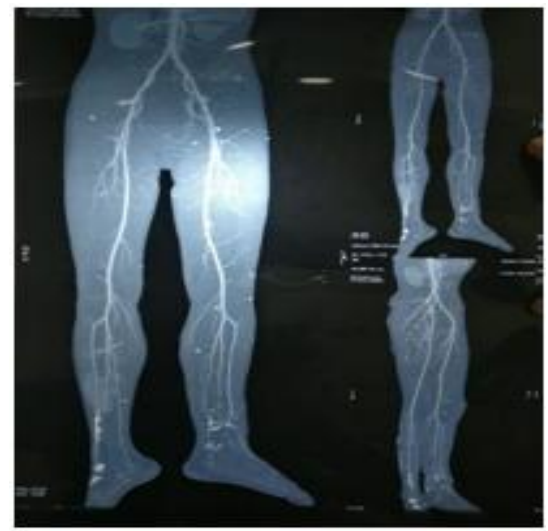

B

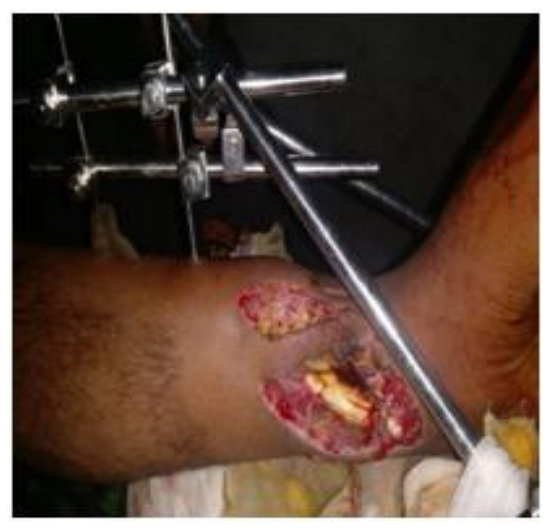

C

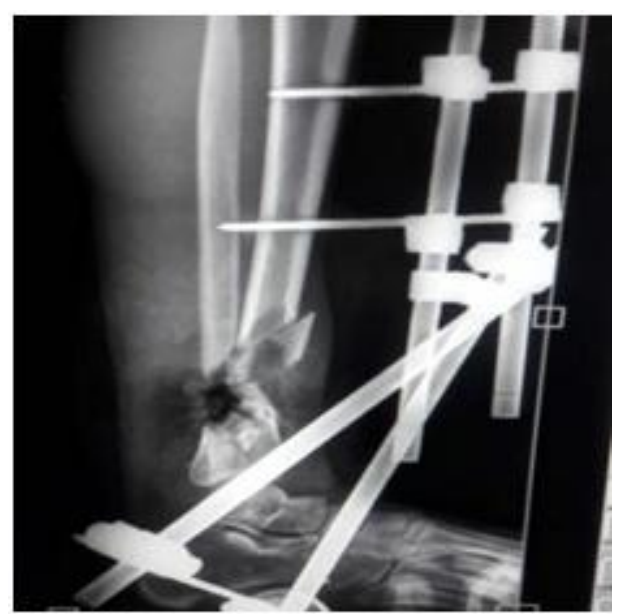

$\mathrm{D}$

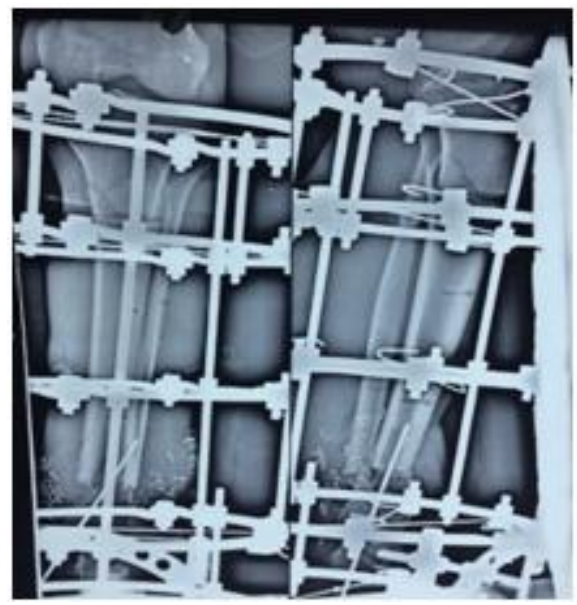

$\mathrm{E}$

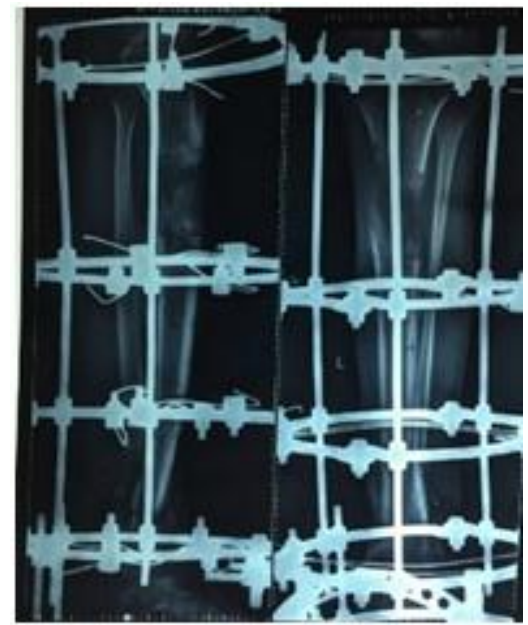

$\mathrm{F}$

Case no. 7. Ty IIIc distal tibia fracture (A) with posterior tibial artery injury (B) managed initially by delta fixator $(\mathrm{C}, \mathrm{D})$, and later converted and maintained in ring fixator (E,); follow-up at 20 weeks $(\mathrm{F})$ 


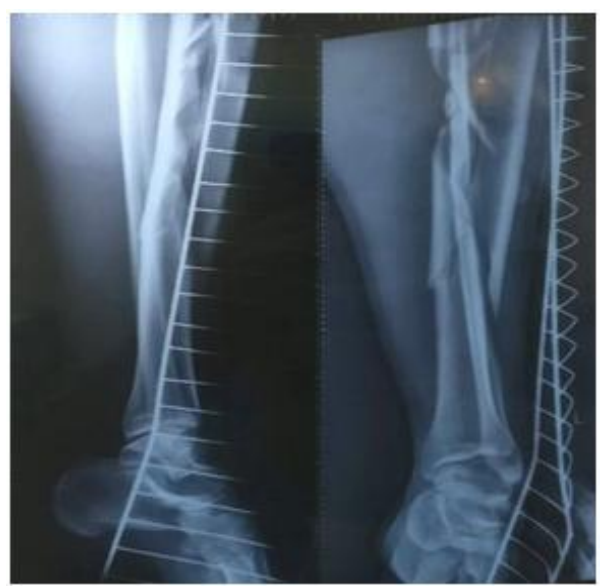

A

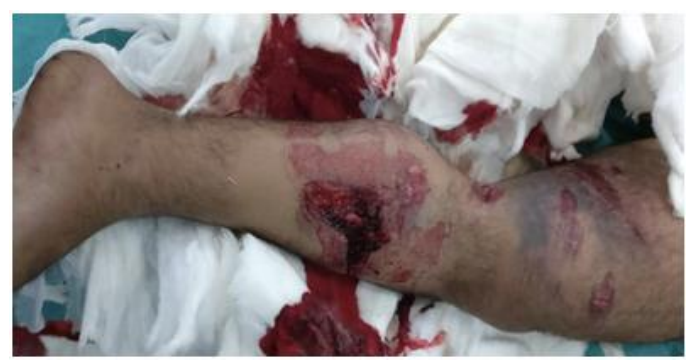

B

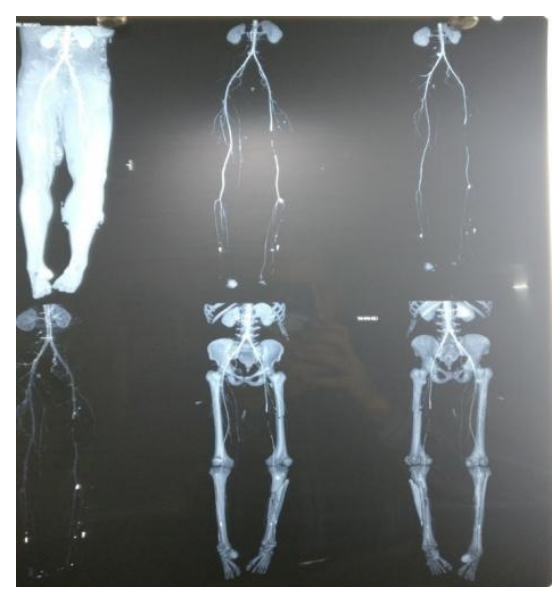

$\mathrm{C}$

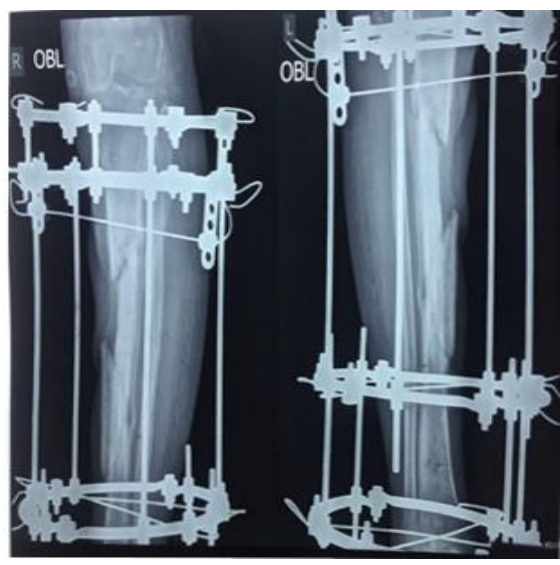

D

Case no. 6. Type IIIc tibia fracture (A, B)with SFA injury (C)initially managed with femoraltibial external fixated and converted at 5 weeks into Taylor Spatial Frame (D)

\section{Complications}

There was no infectious complication reported in the eight cases that underwent early conversion. Mean conversion time in these cases was 5.12 days (range : 3 to 7 days). Case no. 5 with type IIIc injury developed gangrene and required below - knee amputation. Case no. 6 which had a TSF applied developed multiple pin-tract infection and required serial debridements and prolonged antibiotic cover. One patient with a compound distal femur fracture fixed with locking plate (Case no. 12) placed after eight weeks of external fixation developed infectious complications. The outcome was favorable after surgical debridement, antibiotic cement beads, and systemic antibiotic therapy allowing the device to be left in place until bone healing.

\section{Union Status}

Bone union was achieved in fourteen patients. One patient needed amputation on the fifth postop day. Case no. 3 was converted from external fixation to ORIF+ bone grafting with reamed interlocking nailing at 13 weeks but showed no evidence of union at 6 months. Dynamisation of nail together with cancellous BG was done at 6 months but did not improve the healing status even at the last follow-up of 15 months. Pertinently this patient was a chronic smoker. Case no. 9 which was converted into ring fixator had no evidence of healing at 6 months. Cancellous bone grafting was used twice in this case but still failed to unite at last follow-up of 20 months. In patients with early conversion to internal fixation, the mean time to bone healing was 2.8 months (range: 2 to 3 months). In the eight cases of prolonged external fixation, the mean time to bone healing was 6.3 months (range: 15 to 17 months).

\section{Functional Recovery}

All patients with fully united fractures were able to walk with full weight bearing. Eight patients with extended treatment returned to work after 1 year. Three patients resumed their original jobs 
while two patients took over more simpler work. Case no. 4 with sciatic nerve injury had foot drop and is still following a rehabilitation protocol. All the eight cases with early conversion returned to work within one year after trauma.

\section{Discussion}

Damage control orthopaedics (DCO) is a staged approach ideal for polytrauma patients with unstable hemodynamic state and associated injuries. Early conversion of external fixators to internal fixation is a favoured treatment plan as this gives best results in terms of bone healing and functional recovery ${ }^{3}$. In our series, the mean time to healing for fractures was shorter after external fixator conversion. Infection remains the main factor influencing the outcome negatively ${ }^{3}$.

The risk of infection is not much different when conversion from external fixation to intramedullary nailing has been performed within two weeks post-trauma compared with primary nailing ${ }^{4}$. Nowotarski et al. in a large series suggested that immediate external fixation followed by early closed intramedullary nailing is a safe treatment method for fractures of the shaft of femur in selected multiply injured patients ${ }^{5}$. Malik et al. in a series of 59 patients concluded that immediate external fixation followed by early closed interlocking nailing is a safe and effective treatment for open fractures of shaft of femur and tibia ${ }^{6}$. Yoshiyasu et al. suggested that immediate IMN resulted in a lower postoperative infection rate than late $\mathrm{IMN}^{7}$.

\section{Prolonged External Fixation}

Michail et al. suggested that unilateral external fixators can be used as primary and definitive treatment strategy for tibia shaft fractures and are associated with a low deep infection rate ${ }^{4}$. Change of the fixator is warranted in case of a delay in callus formation. Continuous external fixation is effective in case of infectious complications as also for management of soft tissue injuries requiring multiple debridements and flap coverage $^{8}$. Conversion to anIlizarov or hybrid fixator and Taylor Spatial Frame is a preferred option for preventing stiffness at knee in case of periarticular fractures, correction of axial deviations or management of bone defects.

Late fixator removal and conversion to ORIF with bone grafting may be required in case of delayed union (five in this series; case no's. 2, 3,8,12,17). One must be vigilant to ensure there is no risk of infection prior to conversion based on clinical, biological and radiological examinations. Labeled leukocyte scintigraphy is also a reliable method to rule out any infective focus. Once the fixator has been removed, pin tract healing should be achieved prior to performing internal fixation ${ }^{9}$.

\section{Conclusion}

Complex limb injuries require a staged treatment protocol. The improvements achieved in plastic and reconstruction surgery allow early conversion to internal fixation in compound tibia fractures. Our study highlights the utility of temporary external fixation as a damage control treatment modality in enhancing bone healing and functional recovery in type III Gustilo-Anderson compound tibia fractures.

\section{Disclosure of interest}

The authors declare that they have no conflicts of interest concerning this article.

\section{References}

1. https://www.aaos.org/search.aspx?id=32\& srchtext $=$ Christina + L. + Boulton $\% 2 c+M D$

2. Anil kumarjoshi, chitrajoshi, mridusingh, et al. Road traffic accidents in hilly regions of northern india: what has to be done?World journal of emergency medicine $5(2,112,2014)$

3. L. Mathieua, F. Bazilea, R. Barthélémya, P. Duhamelb, et al. Damage control orthopaedics in the context of battlefield injuries: The use of temporary external fixation on combat trauma soldiers Orthopaedics \& Traumatology: Surgery \& Research (2011) 97, 852—859 
4. Beltsios, M., Savvidou, O., Kovanis, J. et al. External fixation as a primary and definitive treatment for tibial diaphyseal fractures. Strat Traum Limb Recon (2009) 4: 81. https://doi.org/10.1007/s11751-0090062-3

5. Nowotarski PJ, Turen $\mathrm{CH}$, Brumback RJ, Scarboro JM.Conversion of External Fixation to Intramedullary Nailing for Fractures of the Shaft of the Femur in Multiply Injured Patients. The Journal of Bone and Joint Surgery 82(6):781-8

6. Malik ZU, Hanif MS, Safdar A, Masood T. Planned external fixation to locked intramedullary nailing conversion for open fractures of shaft of femur and tibia.J Coll Physicians Surg Pak. 2005 Mar;15(3):1336.

7. Yoshiyasu Uchiyama, Yuka Kobayashi, Gro Ebihara, Kosuke Hamahashi, Masahiko Watanabe. Retrospective comparison of postoperative infection and bone union between late and immediate intramedullary nailing of Gustilo grades I, II, and IIIA open tibial shaft fractures. Trauma Surg Acute Care Open 2016;1:14. doi:10.1136/tsaco-2016-00003

8. Nicoll EA. Closed and open management of tibial fractures. Clin Orthop Relat Res 1974:144-53. [PubMed] [Google Scholar]

9. Yokoyama K, Uchino M, Nakamura K, Ohtsuka H, Suzuki T, Boku T, Itoman M. Risk factors for deep infection in secondary intramedullary nailing after external fixation for open tibial fractures. Injury 2006;37:554-60. doi:10.1016/j.injury.2005.08.026

[PubMed] [Google Scholar]. 